\title{
Clinical subtypes of breast cancer in Thai women: a population-based study of Chiang Mai province
}

\author{
Patumrat Sripan ${ }^{1,2,3}$, Hutcha Sriplung ${ }^{4}$, Donsuk Pongnikorn 5 , Surichai Bilheem ${ }^{4}$, Shama Virani ${ }^{4}$, \\ Narate Waisri², Chirapong Hanpragopsuk², Puttachart Maneesai², Panrada Tansiri², \\ Imjai Chitapanarux ${ }^{1,2,6, *}$
}

Abstract

Background: The change over time of distribution of breast cancer subtypes using population-based data has not been reported.

Objective: To describe the change over time of the distribution of female breast cancer by clinical subtype among the population in Chiang Mai, Thailand.

Methods: Data of breast cancer patients from Chiang Mai Cancer Registry, diagnosed from 2004 to 2013 were combined with immunohistochemical status from medical record, and used to describe the proportions of clinical breast cancer subtypes: (1) luminal A-like (ER+/PR+ and HER2-), (2) luminal B-like (ER+/PR+ and HER2+), (3) HER2 (ER- and PR- and HER2+), (4) triple-negative (ER- and PR- and HER2-). The distribution of breast cancer subtypes by age group was also described.

Results: Among 3,228 female breast cancer cases diagnosed during 2004-2013, the median age was 52 years and most patients presented at the regional stage. The unknown tumor subtype was lower than $25 \%$ in the periods 2008 2009, 2010-2011, and 2012-2013. In those periods, the proportions of luminal A-like were 33\%, 36\%, and 48\%; the proportions of luminal B-like were $14 \%, 20 \%$, and $16 \%$, the proportions of HER 2 were $15 \%, 14 \%$, and $13 \%$; and the proportions of triple-negative were $16 \%, 14 \%$, and $13 \%$, respectively. In comparison with other groups, women aged $\geq 60$ years had a significantly higher proportion of luminal A-like $(P=0.001)$, while women aged $<40$ years tended to have a higher proportion of triple-negative $(P=0.10)$.

Conclusions: The proportion of breast cancer with luminal subtypes is increasing. Thus, in the future, treatment protocols with a variety of hormone therapies should be provided in order to improve efficacy and coverage of treatment for this population.

Keywords: age groups; breast neoplasms; women

\footnotetext{
*Correspondence to: Imjai Chitapanarux, Department of Radiology, Faculty of Medicine, Chiang Mai University, Chiang Mai 50200, Thailand, e-mail: imjai.chitapanarux@cmu.ac.th

'Northern Thai Research Group of Radiation Oncology (NTRG-RO), Faculty of Medicine, Chiang Mai University, Chiang Mai 50200, Thailand ${ }^{2}$ Chiang Mai Cancer Registry, Faculty of Medicine, Chiang Mai University, Chiang Mai 50200, Thailand

${ }^{3}$ Research Institute for Health Sciences, Chiang Mai University, Chiang Mai 50200, Thailand

${ }^{4}$ Epidemiology Unit, Faculty of Medicine, Prince of Songkla University, Songkhla 90110, Thailand

${ }^{5}$ Cancer Registry Unit, Lampang Cancer Hospital, Lampang 52000, Thailand

${ }^{6}$ Division of Radiation Oncology, Department of Radiology, Faculty of Medicine, Chiang Mai University, Chiang Mai 50200, Thailand
}

¿ Open Access. ( 2019 Patumrat Sripan et al., published by Sciendo. (c) BY-NC-ND This work is licensed under the Creative Commons Attribution NonCommercial-NoDerivatives 4.0 License. 
Breast cancer is the most common cancer among women globally [1]. There were approximately 1.7 million reported cases of breast cancer and 0.5 million deaths in women worldwide according to the GLOBOCAN 2012 released by the International Agency of Research on Cancer (IARC) [2]. The agestandardized incidence rate (ASR) was 28.5 per 100,000 in Thai women based on data collected during 2010-2012 [3]. In northern Thailand, the incidence of breast cancer continues to increase [4]. Similarly, in the Chiang Mai population, our previous study showed that female breast cancer incidence rates increased from an ASR of 14.8 per 100,000 women-years in 1989 to 32.9 cases per 100,000 women-years in 2013. The incidence rate of female breast cancer increased at an APC of $3.1 \%$ (95\% confidence interval $[\mathrm{CI}]: 2.6 \%, 3.7 \%)$. From projections, we found that the highest incidence might reach an ASR of 36.7 per 100,000 women-years in the next decade and the incidence in elderly females tend to be higher than the middle-aged females [5]. Breast cancer was responsible for nearly 5,092 deaths among Thai women in 2012 [6]. The clinical subtype of the breast cancer has become an important consideration in treatment decision making. In conjunction with conventional clinical factors such as tumor size and grade, lymph node involvement and surgical margins, the molecular classification of the tumor provides prognostic information for the heterogeneous disease of breast cancer [7].

Clinical subtype distribution has varied in different age groups, luminal-A subtype was likely to have higher proportion in the elderly group compared to other groups. Whereas, the proportion of triple-negative was highest in young age group compared to other groups $[8,9]$. Aging plays an important role on incidence and also the efficacy of treatment. Clinical subtype of breast cancer by age group may be useful for treatment and facility provision.

A number of hospital-based studies have reported the proportion of breast cancer subtypes based on estrogen receptor (ER)/progesterone receptor (PR) and/or human epidermal growth factor receptor 2 (HER2) status in Thai women [10 12]. However, the change over time of distribution of breast cancer subtypes using population-based data has not been reported. Therefore, our study describes the distribution of breast cancer subtypes over the period from 2004 to 2013 in the Chiang Mai population in Northern Thailand.

\section{Materials and methods}

The data used in this study are retrospective data from the cancer registry and the study was approved by the Research Ethics Committee of the Faculty of Medicine, Chiang Mai University (certificate of approval no. 316/2016).
This observational study extracted data of women diagnosed with breast cancer during 2004-2013 and living in Chiang Mai province were collected by the Chiang Mai Cancer Registry using International Classification of Diseases-10th edition (ICD-10) codes C50. The Chiang Mai Cancer Registry is a population-based cancer registry operating in the Maharaj Nakorn Chiang Mai Hospital, Faculty of Medicine, Chiang Mai University. The data were collected from all hospitals in Chiang Mai, including Maharaj Nakorn Chiang Mai Hospital, which is a university hospital, as well as government hospitals, municipal hospitals, private hospitals, and community hospitals. Patient data were systematically collected and carefully verified by cancer registry personnel and using cancer registry software (CanReg5). Cancer registry data included patient profiles, such as date of birth, age at diagnosis, clinical diagnosis, pathological report, clinical extent of disease before treatment, and initial treatment. Immunohistochemical (IHC) status including ER, PR, and HER2 was extracted from the medical record.

The stage of breast cancer was categorized based on the extent of disease of cancer. Localized tumors are restricted to the breast, regional tumors involve axillary lymph nodes, and distant stage involves spreading of cancer to other parts of the body.

\section{Classification of clinical breast cancer subtype}

ER, PR count as positive when the percentage of cells with nuclear positivity was found at least $1 \%$ and HER 2 count as positive when the percentage of cells with uniform intense complete membrane staining was found at least $1 \%$. The four clinical subtypes are based on the presence or absence of molecular markers, including ER, PR, and HER2 [13, 14]. Luminal A-like is characterized by ER-positive $(+)$ and/or $\mathrm{PR}+$ and HER2 negative (-) status. Luminal B-like subtype is distinguished by ER+ and/or PR+ and HER2+ status. The HER2 subtype is characterized by HER2+, ER-, and PR-. Triple-negative subtypes are negative for all three markers (ER-, PR-, and HER2-).

\section{Statistical analysis}

The proportions of clinical subtype of breast cancer were described in 2-year periods from 2004 to 2013. Trend of proportion was examined using $\chi^{2}$ test for trend in proportion and trend of median age were examined using Mann-Kendall trend test. Frequency distributions of breast cancer subtypes by age, stage at diagnosis, and diagnosis year were calculated. A $\chi^{2}$ test was used to compare distributions using Stata version 11 (StataCorp LP, College Station, TX, USA). 
Analyses were conducted for all females, young age group ( $<40$ years), middle-aged group ( $40-59$ years), and elderly group ( $\geq 60$ years) to determine the distribution of breast cancer subtype by age group.

Visually illustrate trends and trends test were conducted using $\mathrm{R}$ program. All reported $P$ are two-sided values and $P<0.05$ was considered statistically significant.

\section{Results}

\section{Distributions of clinical subtypes by patient characteristics}

Among the 3,228 female breast cancer cases with an overall median age of 52 years (interquartile range, IQR: 45-60) diagnosed during 2004-2013, 25\%, 66\%, 7\%, and 2\% had localized stage, regional stage distant stage, and unknown stage, respectively, at diagnosis. Table 1 shows the characteristics of patients at diagnosis over time (2004-2013). In women with known subtypes, the distributions of clinical subtypes were significantly different by age group, stage, and year of diagnosis (Table 2).

\section{Change of proportion of breast cancer subtypes over time}

In 2-year period, the proportion of unknown tumor subtype dramatically declined from $65 \%$ in $2004-2005$ to $9 \%$ in
2012-2013. In the most recent three periods, 2008-2009, 2010-2011, and 2012-2013, in which the unknown tumor subtype was lower than $25 \%$, the proportion of luminal A-like was 33\%, 36\%, and $48 \%(P<0.001)$; the proportion of luminal B-like was $14 \%, 20 \%$, and $16 \%(P=0.2)$; the proportion of HER2 was $15 \%, 14 \%$, and $13 \%(0.24)$; and the proportion of triple-negative was $16 \%, 14 \%$, and $13 \%(0.11)$, respectively (Figure 1).

\section{Proportion of breast cancer subtypes by age group}

Among the 2,340 breast cancer patients with known clinical subtype, the most common subtype found in all age groups was luminal A-like. Overall, the proportion of luminal A-like, luminal B-like, HER2, and triple-negative was $46 \%, 20 \%$, $16 \%$, and $18 \%$, respectively. The elderly group had a significantly higher proportion of luminal A-like (52\%) compared to the other groups: $45 \%$ in the middle-aged group and $38 \%$ in the young age group $(P=0.001)$. The proportion of triple-negative was higher in the young age group $(22 \%)$ than in other groups: $17 \%$ in the middle-aged group and $19 \%$ in the elderly group, but this was not significantly different $(P=0.10)$. The proportions of luminal B-like were $26 \%$, $20 \%$, and $16 \%$ in the young age, middle-aged, and elderly groups, respectively. The proportions of HER2 tumors were $14 \%, 18 \%$, and $13 \%$ in the young age, middle-aged, and elderly groups (Table 1).

Table 1. Characteristics of patients at diagnosis over time (2004-2013)

\begin{tabular}{|c|c|c|c|c|c|c|c|}
\hline & \multirow[t]{2}{*}{ Total, $n$ (\%) } & \multicolumn{5}{|c|}{ Year of diagnosis } & \multirow[t]{2}{*}{$P^{a}$} \\
\hline & & 2004-2005 & 2006-2007 & 2008-2009 & 2010-2011 & 2012-2013 & \\
\hline Number of patients & $3,228(100)$ & $530(17)$ & $571(18)$ & $668(21)$ & $656(20)$ & $803(25)$ & \\
\hline \multicolumn{8}{|l|}{ Age (years) } \\
\hline Median (IQR) & $45(52-60)$ & $50(44-58)$ & $51(44-59)$ & $51(45-58)$ & $53(46-60)$ & $54(47-61)$ & $0.043^{b}$ \\
\hline$<40$ & $349(11)$ & $63(12)$ & $76(13)$ & $67(10)$ & $72(11)$ & $71(9)$ & 0.02 \\
\hline $40-59$ & $2,072(64)$ & $346(65)$ & $358(63)$ & $465(70)$ & $401(61)$ & $502(62)$ & 0.20 \\
\hline$\geq 60$ & $807(25)$ & $121(23)$ & $137(24)$ & $136(20)$ & $183(28)$ & $230(29)$ & 0.002 \\
\hline \multicolumn{8}{|l|}{ Stage } \\
\hline Localized & $794(25)$ & $125(24)$ & $132(23)$ & $156(23)$ & $155(24)$ & $226(28)$ & 0.04 \\
\hline Regional & $2,131(66)$ & $352(66)$ & $390(68)$ & $462(69)$ & $447(68)$ & $480(60)$ & 0.02 \\
\hline Distant & $235(7)$ & $38(7)$ & $39(7)$ & $44(7)$ & $49(8)$ & $65(8)$ & 0.39 \\
\hline Unknown & $68(2)$ & $15(3)$ & $10(2)$ & $6(1)$ & $5(1)$ & $32(4)$ & 0.17 \\
\hline
\end{tabular}

$\mathrm{IQR}$, Interquartile range.

${ }^{\mathrm{a}} \chi^{2}$ test for trend in proportions.

${ }^{\mathrm{b}}$ Mann-Kendall trend test. 
Table 2. Distribution of breast cancer subtypes by age, stage and year of diagnosis (2004-2013)

\begin{tabular}{|c|c|c|c|c|c|}
\hline \multirow[t]{2}{*}{ Number of patients (\%) } & \multicolumn{4}{|c|}{ Subtypes } & \multirow[t]{2}{*}{$P^{a}$} \\
\hline & $\begin{array}{c}\text { Luminal A-like } \\
1,074(46)\end{array}$ & $\begin{array}{c}\text { Luminal B-like } \\
469(20)\end{array}$ & $\begin{array}{c}\text { HER2 } \\
375(16)\end{array}$ & $\begin{array}{c}\text { Triple-negative } \\
422(18)\end{array}$ & \\
\hline Age (years) & & & & & $<0.001$ \\
\hline$<40$ & 97 (38\%) & $66(26 \%)$ & $36(14 \%)$ & $57(22 \%)$ & \\
\hline $40-59$ & $681(45 \%)$ & 309 (20\%) & $264(18 \%)$ & $256(17 \%)$ & \\
\hline$\geq 60$ & $296(52 \%)$ & $94(16 \%)$ & $75(13 \%)$ & 109 (19\%) & \\
\hline Stage & & & & & $<0.001$ \\
\hline Localized & $351(55 \%)$ & $100(16 \%)$ & $81(13 \%)$ & $100(16 \%)$ & \\
\hline Regional & $636(42 \%)$ & $334(22 \%)$ & $253(17 \%)$ & 297 (19\%) & \\
\hline Distant & $72(44 \%)$ & $31(19 \%)$ & $38(23 \%)$ & $24(14 \%)$ & \\
\hline Unknown & $15(65 \%)$ & $4(17 \%)$ & $3(13 \%)$ & $1(5 \%)$ & \\
\hline Year of diagnosis & & & & & $<0.001$ \\
\hline 2004 & $35(38 \%)$ & $22(24 \%)$ & $19(21 \%)$ & $15(17 \%)$ & \\
\hline 2005 & $32(33 \%)$ & $20(21 \%)$ & $20(21 \%)$ & $24(25 \%)$ & \\
\hline 2006 & $76(45 \%)$ & $35(21 \%)$ & $20(12 \%)$ & $38(22 \%)$ & \\
\hline 2007 & $93(48 \%)$ & 37 (19\%) & $24(13 \%)$ & $38(20 \%)$ & \\
\hline 2008 & $128(50 \%)$ & $35(14 \%)$ & 40 (16\%) & $53(20 \%)$ & \\
\hline 2009 & $91(34 \%)$ & $56(21 \%)$ & $60(23 \%)$ & $57(22 \%)$ & \\
\hline 2010 & 109 (44\%) & $55(22 \%)$ & $48(19 \%)$ & $38(15 \%)$ & \\
\hline 2011 & $124(42 \%)$ & 77 (26\%) & 41 (14\%) & 51 (18\%) & \\
\hline 2012 & 175 (51\%) & 77 (22\%) & 50 (14\%) & 45 (13\%) & \\
\hline 2013 & $211(55 \%)$ & 55 (14\%) & 53 (14\%) & 63 (17\%) & \\
\hline
\end{tabular}

${ }^{\mathrm{a}} \chi^{2}$ test.

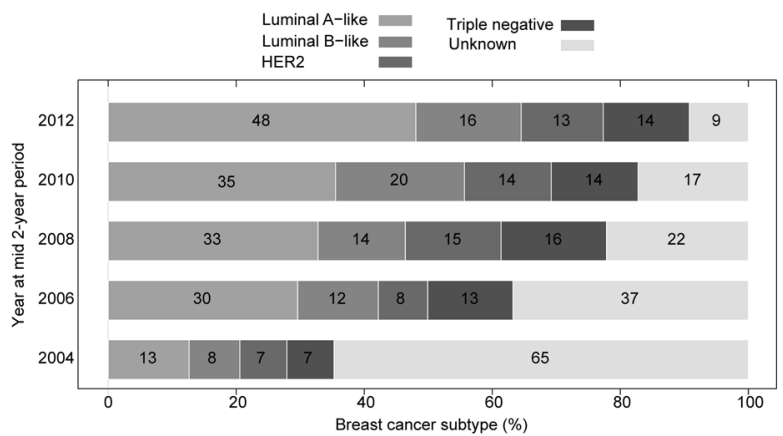

Figure 1. Distribution of breast cancer subtypes in all age groups over 2-year period from 2004 to 2013

\section{Discussion}

According to the population-based cancer registry data for the Chiang Mai population in 2004-2013, the incidence of breast cancer significantly increased [5]. While the proportion of unknown subtype has been decreasing, the proportion of luminal subtype (ER+ and/or PR+) has been increasing, particularly the proportion of luminal A-like (ER+ or PR+ and HER2-). The proportion of HER2 (ER- and PR- and HER2+) subtypes and triple-negative subtypes (ER- and PRand HER2-) were stable and lower than $20 \%$.

Only a few studies previously reported on the proportion of breast cancer subtypes based on ER/PR and HER2 in the Thai population.

First, a study of patients with invasive ductal carcinomas in Siriraj Hospital reported the proportion of female breast cancer clinical subtypes [10]. Compared to the Siriraj Hospital study, which assessed 324 breast cancer patients diagnosed during 2009-2010, our study found a lower proportion of luminal A-like in our population in 2009-2010 (39\% in our study vs. $59 \%$ in the Siriraj study), but a higher proportion of luminal B-like and HER2 (22\% and 21\%, respectively, in our study vs. $12 \%$ and $13 \%$, respectively, in the Siriraj Hospital study). However, the proportion of triple-negative in our population was similar to that reported in the Siriraj Hospital study, $18 \%$ vs $15 \%$. 
Second, a study of the northeastern Thailand population on 294 patients diagnosed with breast cancer at Srinagarind Hospital [12] from January 2004 to June 2006 found that the proportion of luminal A-like (ER+ or PR+ and HER2-), luminal B-like (ER+ or PR+ and HER2+), HER2 (ER- and PR - and HER2+) and triple-negative was $51 \%, 8 \%, 19 \%$, and $22 \%$, respectively. Compared to the Srinagarind Hospital study, our study found a slightly lower proportion of luminal A-like ( $40 \%$ vs. $51 \%$ ) but a significantly higher proportion of luminal B-like ( $22 \%$ vs. $8 \%)$. The proportions of HER2 and Triple-negative in our study ( $17 \%$ and $22 \%$, respectively) were similar to that in the Srinagarind Hospital study.

The most recent study in southern Thailand [15] reported, $46 \%, 28 \%, 13 \%$, and $13 \%$, respectively, of the subtypes luminal A-like, luminal B-like, HER2, and triple-negative subtypes among 635 female breast cancer patients for whom clinical subtype information was available in 2010-2012. The proportion of luminal A-like in our study was the same as the southern Thailand study. The proportion of luminal B-like was lower in our study (24\%), while the proportions of HER2 and triple-negative in our study ( $16 \%$ and $15 \%$, respectively) were slightly higher than the southern Thailand study. A Westernbased population study using data of female breast cancer cases diagnosed during 1999-2003 in California, showed a $12 \%$ proportion of triple-negative tumors. Women with triplenegative breast cancers were significantly more likely to be under age 40 [8]. Another larger study based on data from 17 population-based cancer registries that participate in the SEER program in the United States reported that among 50,571 cases in patients diagnosed in 2010, 73\% were luminal A-like (HR+/ HER $2-$ ), $12 \%$ were triple-negative (HR-/HER2-), $10 \%$ were luminal B-like (HR+/HER2+), and 5\% were HER2 (HR-/ HER2+). This study also showed that the proportion of triplenegative tumors was significantly higher in women age $<65$ [9]. The distribution of female breast cancer subtypes in the US population and our Chiang Mai population are different. The proportion of luminal A-like was much higher and HER2 was moderately lower in the US population compared to our Chiang Mai population, but the distribution of our population was likely closer to other studies among the Thai population $[10,12,15]$, despite that these Thai studies were not population-based study and were conducted in different parts of Thailand. Therefore, the distributions of female breast cancer by subtypes using population-based data in different parts of Thailand are recommended.

As reported in several studies [8,9], our study found similar results that the most common subtype in all age groups was luminal A-like, which was likely to have a higher proportion in the elderly group compared to other groups. Our previous study showed a significant increase of breast cancer incidence in the elderly [6]; in the present study, we also found the increase of median age over time (Table 1). This might explain the larger proportion of luminal A-like subtype over time (Figure 1).

Moreover, an association between mammographic screening and detection of breast cancer with hormone positive, that is, ER+ has been shown $[16,17]$. In the present study, percentage of localized tumor has been significantly increased $(P=0.04)$ and may represent the increased coverage of screening. Although organized mammographic screening has not been established in Thailand, the percentage of coverage increased in different regions of the country. According to the National Health and Welfare Survey in 2007 and the Reproductive Health Survey in 2009 [18], the screening coverage was found to be stable in Bangkok (13\%) with moderate increase in the North, Northeast, and South (from $4 \%$ to $9 \%, 5 \%$ to $9 \%$, and $7 \%$ to $10 \%$, respectively). The coverage of mammographic screening was highest in Bangkok, followed by the South region [18]. This might explain the higher proportion of luminal subtypes in Bangkok and the South compared to our Chiang Mai population, but our results were similar to what was found in the Northeast in the corresponding period of study.

IHC analyses including ER, PR, HER2, and Ki-67, define the luminal A, luminal B, HER2, and Basal-like subtypes [19]. However, our study only used ER, PR, and HER2 status to classify clinical subtype of breast cancer because Ki-67 information is not routinely collected and was unavailable. Therefore, we used the available IHC status to approximate the breast cancer subtype into the four subtypes: luminal A-like, luminal B-like, HER2, and triple-negative. However, we found this distribution of approximated subtypes of breast cancer using IHC was similar to what was reported in an Asian-population study that was based on molecular data [20], in which the luminal subtypes (luminal A+luminal B), HER2, and triple-negative subtypes were $65 \%, 14 \%$, and $13 \%$, respectively. Thus, this IHC classification may be more precise in distinguishing breast cancer subtypes if the luminal A-like and luminal B-like subtypes are merged into a single luminal subtype.

At the beginning period of our study, HER2 status that was necessary for IHC classification was not routinely examined. Therefore, clinical subtypes were not able to classify in such periods in this population. This can lead to an underestimation of the proportion of clinical subtypes. However, age distribution of unknown subtype was not significantly different from known subtype $(P=0.6)$. Fortunately, in recent years, subtypes of breast cancer have been classified more accurately with less unknown subtype. The change 
of subtype distribution overtime could be more accurately determined to observe the most three recent periods, which had $<25 \%$ unknown subtype.

Moreover, our study has lack of tumour, node, metastasis (TNM) staging data defined by American Joint Committee on Cancer (AJCC) because of incomplete staging from some sources of data. The extent of disease which is commonly applied for population-based cancer registry data was therefore used instead.

Another limitation of our study is the delay of data collection. These are population-based data with active method of collection which has delay time around 2 years. Some additional time was spent for IHC status review of all individual record from all hospitals in Chiang Mai province because these data were not routinely collected by the cancer registry. Inclusion of these essential clinical data in the cancer registry would be encouraged for less time delay. However, our study is the first population-based study that describes the proportions of female breast cancer by clinical subtype and the change over time of those proportions in the Thai population. Moreover, this population-based data were collected by the active method, so we consider the data to be generally high quality, with more than $95 \%$ having histology verification $(\% \mathrm{HV})$ and $<1 \%$ is death certificate only cases (\%DCO).

As found in the results of hospital-based studies [10, 12, 15], the proportions of clinical subtypes of breast cancer were different in different parts of Thailand. Thus, the trends in incidence of breast cancer by clinical subtypes are likely to be different between sets of regions. In the future, when the classification of clinical subtype of breast cancer could become more stably accurate, the incidence estimation of female breast cancer by clinical subtypes using population-based data from different parts of Thailand is encouraged to determine the distribution of clinical subtype nationally and sub-nationally. The results of our study may guide for the better treatment plan considering the breast cancer subtypes. Since the proportion of hormone positive subtypes has been increasing, the inclusion of treatment facilities with a variety of hormone therapies as essential medicines for universal health coverage should be considered.

\section{Conclusion}

In the last decade, increased ability to identify subtypes has led to better treatment planning. Although the proportion of breast cancer with HER2 and triple-negative subtypes has remained stable at $<20 \%$ in our population, anti-HER 2 therapies should be made available. The proportion of hormone positive subtypes, luminal A-like, and luminal B-like has been increasing. Therefore, in the future, treatment facilities with a variety of hormone therapies should be provided in order to improve the efficacy and coverage of treatment for this population.

Author contributions. PS, HS, DP, SB, SV, and IC contributed substantially to the conception and design of this study. NW, CH, PN, PT, and IC contributed substantially to the acquisition of data. PS and SB analyzed and interpreted the data. PS, HS, and IC drafted the manuscript. DP, SB, SV, NW, $\mathrm{CH}, \mathrm{PM}, \mathrm{PT}$, and IC contributed substantially to its critical revision. All the authors approved the final version submitted for publication and take responsibility for the statements made in the published article.

Acknowledgment. We would like to thank all the medical staff members of the Faculty of Medicine Chiang Mai University in particular Malisa Poungsombat, Varunee Khamsan, and Aumnart Mawoot for their assistance on data collection, and all the physicians both government and private hospitals in Chiang Mai Province, for their collaboration. We also wish to thank the Bureau of Registration Administration, Ministry of Interior for providing death certificate data for cancer registry. This study was supported by research funding from Faculty of Medicine, Chiang Mai University. The data sets used and/ or analyzed during the current study are available from the corresponding author on reasonable request.

Conflict of interest statement. The authors have completed and submitted the ICMJE Uniform Disclosure Form for Potential Conflicts of Interest. None of the authors disclose any conflict of interest.

\section{References}

[1] Stewart BW, Wild CP, editors. World Cancer Report 2014. IARC Press. 2014

[2] Ferlay J, Soerjomataram I, Ervik M, Dikshit R, Eser S, Mathers C, et al. GLOBOCAN: cancer incidence and mortality worldwide: IARC cancer base no. 11. International Agency for Research on Cancer. 2013.

[3] Imsamran W, Chaiwerawattana A, Wiangnon S, Pongnikorn D, Suwanrungruang K, Sangrajrang S, et al. Cancer in Thailand Volume VIII, 2010-2012, National Cancer Institute, Ministry of Public Health; Bangkok, Thailand. 2015.

[4] Pongnikorn D, Daoprasert K, Waisri N, Laversanne M, Bray F. Cancer incidence in northern Thailand: results from six 
population-based cancer registries 1993-2012. Int J Cancer. 2018; 142:1767-75. doi:10.1002/ijc.31203.

[5] Sripan P, Sriplung H, Pongnikorn D, Virani S, Bilheem S, Chaisaengkhaum $\mathrm{U}$, et al. Trends in female breast cancer by age group in the Chiang Mai population. Asian Pac J Cancer Prev. 2017; 18:1411-6. doi:10.22034/APJCP.2017.18.5.1411.

[6] Youlden DR, Cramb SM, Yip CH, Baade PD. Incidence and mortality of female breast cancer in the Asia-Pacific region. Cancer Biol Med. 2014; 11:101-15. doi:10.7497/j.issn.20953941.2014.02.005.

[7] Haque R, Ahmed SA, Inzhakova G, Shi J, Avila C, Polikoff J, et al. Impact of breast cancer subtypes and treatment on survival: an analysis spanning two decades. Cancer Epidemiol Biomarkers Prev. 2012; 21: 1848-55. doi:10.1158/1055-9965.EPI-12-0474.

[8] Bauer KR, Brown M, Cress RD, Parise CA, Caggiano V. Descriptive analysis of estrogen receptor (ER)-negative, progesterone receptor (PR)-negative, and HER2-negative invasive breast cancer, the so-called triple-negative phenotype: a population-based study from the California Cancer Registry. Cancer. 2007; 109:1721-8. doi:10.1002/ cncr.22618.

[9] Howlader N, Altekruse SF, Li CI, Chen VW, Clarke CA, Ries LA, et al. US incidence of breast cancer subtypes defined by joint hormone receptor and HER2 status. J Natl Cancer Inst. 2014; 106. doi:10.1093/ jnci/dju055.

[10] Chuthapisith S, Permsapaya W, Warnnissorn M, Akewanlop C, Sirivatanauksorn V, Prasarttong Osoth P. Breast cancer subtypes identified by the ER, PR and HER-2 status in Thai women. Asian Pac J Cancer Prev. 2012; 13:459-62.

[11] Lertsanguansinchai P, Chottetanaprasith T, Chatamra K, Sampatanukul P, Wannakrairot P, Rojpornpradit P, et al. Estrogen and progesterone receptors status in Thai female breast cancer patients: an analysis of 399 cases at King Chulalongkorn Memorial Hospital. J Med Assoc Thai. 2002; 85 Suppl 1:S193-202.
[12] Koonmee S, Sirithunyaporn S, Phanomsri E. Hormonal receptors status of breast cancer in northeastern Thai women: an analysis of 241 cases at Srinagarind Hospital. Srinagarind Med J. 2006; 21:343-51.

[13] Brenton JD, Carey LA, Ahmed AA, Caldas C. Molecular classification and molecular forecasting of breast cancer: ready for clinical application? J Clin Oncol. 2005; 23:7350-60.

[14] Nguyen PL, Taghian AG, Katz MS, Niemierko A, Abi Raad RF, Boon WL, et al. Breast cancer subtype approximated by estrogen receptor, progesterone receptor, and HER-2 is associated with local and distant recurrence after breast-conserving therapy. J Clin Oncol. 2008; 26:2373-8.

[15] Virani S, Wetzel EC, Laohawiriyakamol S. Ethnic disparity in breast cancer survival in southern Thai women. Cancer Epidemiol. 2018; 54:82-9. doi:10.1016/j.canep.2018.02.007.

[16] Glass AG, Lacey JV, Carreon JD, Hoover RN. Breast cancer incidence, 1980-2006: combined roles of menopausal hormone therapy, screening mammography, and estrogen receptor status. J Natl Cancer Inst. 2007; 99:1152-61. doi:10.1093/jnci/djn077.

[17] Porter PL, El-Bastawissi AY, Mandelson MT, Lin MG, Khalid N, Watney EA, et al. Breast tumor characteristics as predictors of mammographic detection: comparison of interval- and screendetected cancers. J Natl Cancer Inst. 1999; 91:2020-8.

[18] Mukem S, Meng Q, Sriplung H, Tangcharoensathien V. Low coverage and disparities of breast and cervical cancer screening in Thai women: analysis of National Representative Household Surveys. Asian Pac J Cancer Prev. 2015; 16:8541-51.

[19] Yersal O, Barutca S. Biological subtypes of breast cancer: prognostic and therapeutic implications. World J Clin Oncol. 2014; 5:412-24. doi:10.5306/wjco.v5.i3.412.

[20] Su Y, Zheng Y, Zheng W, Gu K, Chen Z, Li G, et al. Distinct distribution and prognostic significance of molecular subtypes of breast cancer in Chinese women: a population-based cohort study. BMC Cancer. 2011; 11:292. doi:10.1186/1471-2407-11-292. 